\title{
Review of: "Preliminary Outcomes Following Application Of The Real-Time Navigation System Combined With Intraoperative Three-Dimensional C- Arm Computed Tomography For Zygomatico-Orbital Fracture Reconstruction"
}

\author{
Chien Tzung Chen ${ }^{1}$ \\ 1 Chang Gung Memorial Hospital
}

Potential competing interests: The author(s) declared that no potential competing interests exist.

This manuscript described that combination of intraoperative navigation and C arm CT to improve the outcome of zygoma and orbital reconstruction in small number series of patients.

The concept of using navigation and intraoperative C arm CT is not new at all. There were already many literatures reporting its outcome.

They reported that two patients required implant adjustment once after the intraoperative 3D C-arm assessment without mention adjustment of implant for zygoma or orbital implants. Besides, only 2 of 15 patients required adjustment cannot convey the importance of intraoperative 3D C-arm.

The only assessed outcome of adequacy of zygomatic reduction and orbital reconstruction without control group cannot convey the merit of intraoperative navigation and C arm CT.

Besides, orbital volume measurement using Brainlab software autosegmentation with fine adjustment is not accurate enough especially in orbital blow out fracture. They also need to provide preop, introp and postoperative volume amount to assess the change in between. 